\title{
ON THE EXISTENCE OF FLAT PLANES IN SPACES OF NON-POSITIVE CURVATURE
}

\author{
MARTIN R. BRIDSON
}

(Communicated by James E. West)

\begin{abstract}
Let $X$ be a proper 1-connected geodesic metric space which is nonpositively curved in the sense that it satisfies Gromov's CAT(0) condition globally. If $X$ is cocompact, then either it is $\delta$-hyperbolic, for some $\delta>0$, or else it contains an isometrically embedded copy of the Euclidean plane; these conditions are mutually exclusive. It follows that if the fundamental group of a compact non-positively curved polyhedron $K$ is not word-hyperbolic, then the universal cover of $K$ contains a flat plane.
\end{abstract}

\section{INTRODUCTION}

In his remarkable essay on hyperbolic groups [G], M. Gromov demonstrated, among many other things, that many of the metric properties of non-positively curved Riemannian manifolds are shared by the larger class of CAT $(0)$ metric spaces (as defined in Section 1 below). For example, it follows from work of Eberlein $[E]$ that a compact non-positively curved Riemannian manifold either has a word-hyperbolic fundamental group or else its universal cover contains an isometrically embedded copy of the Euclidean plane; the corresponding result for polyhedral spaces of non-positive curvature is given on page 119 of [G]. Many of Gromov's results in this area have been the subject of lucid exposition by a number of authors, but a detailed account of this useful theorem has not been forthcoming. The main purpose of this note is to give such an account by proving the following result, interesting consequences of which have recently been obtained by Floyd and Parry [FP] and by Mosher [M].

Theorem A. Let $X$ be a proper geodesic metric space which satisfies the $C A T(0)$ inequality globally. If $X$ is cocompact, then the following conditions are equivalent:

(1) There does not exist an isometric embedding of the Euclidean plane into $X$.

(2) $X$ is a visibility space.

(3) $X$ is $\delta$-hyperbolic for some $\delta>0$.

Received by the editors February 23, 1993.

1991 Mathematics Subject Classification. Primary 53C23, 53C42, $20 \mathrm{~F} 32$.

Partially supported by NSF grant DMS-9203500 and FNRS (Suisse). 
Corollary. Let $X$ be a proper geodesic metric space which satisfies the $\mathrm{CAT}(0)$ inequality globally, and let $\Gamma$ be a finitely generated group which acts properly and cocompactly by isometries on $X$. Then $\Gamma$ is word-hyperbolic if and only if there does not exist an isometric embedding of the Euclidean plane into $X$.

This corollary is immediate because being hyperbolic is a quasi-isometry invariant for geodesic metric spaces [G] and if a group acts properly and cocompactly by isometries on a proper geodesic metric space $X$, then its Cayley graph is quasi-isometric to $X$ (see, e.g., [GH]). It remains an open question as to whether or not every word-hyperbolic group arises in this geometric setting. It also remains an open question as to whether or not, with the above notation, the existence of a flat plane in $X$ implies the existence of a free abelian subgroup of rank 2 in $\Gamma$. Indeed this question is even open in the case where $X$ is a Riemannian manifold of non-positive curvature and is also open in the case where $X$ is a piecewise flat 2-complex.

The different conditions appearing in the statement of the above theorem are defined as follows. To say that $X$ is cocompact means that there exists a compact set $K \subseteq X$ such that the translates of $K$ by the full isometry group Isom $(X)$ cover $X$. For example, $X$ is cocompact if it is homogeneous or if it is the universal cover of a compact space such that the group of deck transformations acts by isometries. A metric space is said to be proper if all of its closed bounded subsets are compact. It is said to be a geodesic space if every pair of points can be joined by a topological arc which is isometric to a closed interval of the real line. $X$ is said to be a visibility space if for every pair of non-asymptotic geodesic rays $c, c^{\prime}:[0, \infty) \rightarrow X$ there exists a constant $D>0$ and a geodesic $\gamma: \mathbb{R} \rightarrow X$ such that $d(\gamma(t), c(t)) \leq D$ and $d\left(\gamma(-t), c^{\prime}(t)\right) \leq D$ for all $t>0$. In $\operatorname{CAT}(0)$ spaces this last condition can be expressed in a number of different ways (see Section 2). Roughly speaking, it is equivalent to the requirement that any geodesic segment in $X$, no matter what its length, subtends a small angle at the eye of a distant observer, as is the case in hyperbolic space for example.

A short appendix to this paper contains a remark concerning the characterisation of word-hyperbolic groups as those which satisfy a strictly sub-quadratic isoperimetric inequality.

\section{Basic facts about CAT(0) SPACES}

In this section we recall the definition of a $\operatorname{CAT}(0)$ space and decribe those elements of the geometry of such spaces which we shall need in order to give a self-contained proof of our main result. Much of the material in this section can be found scattered through the literature (e.g. [Ba], [Br], [BGS]). A complete and systematic treatment will be given in our forthcoming book [BH], where the reader will find a more detailed proof of all assertions appearing below.

Definitions. A metric space $(X, d)$ is said to be a geodesic metric space if every pair of points in $X$ can be joined by a topological arc which, with the metric induced by $d$, is isometric to a compact interval of the real line. A geodesic segment in $X$ is a unit speed parametrisation of such an arc. A geodesic triangle $\Delta$ in $X$ is a triple of points (vertices) $p, q, r \in X$ together with a choice of three geodesic segments (sides), one joining each pair of vertices. A comparison 
triangle for $\Delta$ is a triangle $\bar{\Delta}$ in the Euclidean plane $\mathbb{E}^{2}$ with vertices $\bar{p}, \bar{q}, \bar{r}$ such that $d(p, q)=d(\bar{p}, \bar{q}), d(q, r)=d(\bar{q}, \bar{r}), d(p, r)=d(\bar{p}, \bar{r})$ (such a Euclidean triangle is unique up to isometry). Given a side of $\Delta$ and a point $x$ on it, there is a unique comparison point $\bar{x}$ on the corresponding side of the comparison triangle with $d(x, e)=d(\bar{x}, \bar{e})$ for each of the endpoints $e$ of the given side. In general, $\bar{x}$ depends on the choice of side as well as the specified point $x$ (as can be seen by considering the case where two sides of $\Delta$ intersect in their interior); $\bar{x}$ is said to be a comparison point for $x$.

Definition of $\operatorname{CAT}(0)$. A geodesic metric space satisfies the $\operatorname{CAT}(0)$ inequality globally (more briefly, " $X$ is a $\operatorname{CAT}(0)$ space") if and only if

$$
d(x, y) \leq d(\bar{x}, \bar{y})
$$

for every geodesic triangle $\Delta$ in $X$, every pair of points $x$ and $y$ on the sides of $\Delta$, and every choice of comparison points $\bar{x}$ and $\bar{y}$. It is easy to show that this condition is equivalent to requiring only that the above inequality hold in the case where $y$ is a vertex of $\Delta$.

Remark. According to a theorem of Topogonov [CE], a simply connected complete Riemannain manifold satisfies $\mathrm{CAT}(0)$ if and only if all of its sectional curvatures are non-positive. The simplest non-manifold example of a CAT(0) space is a connected simplicial tree endowed with a metric such that each edge is isometric to a compact interval of the real line.

We note the following basic facts about the geometry of geodesics in CAT( 0$)$ spaces.

Lemma 1.1. Let $X$ be a $\mathrm{CAT}(0)$ space.

(1) There is a unique geodesic segment joining every pair of points in $X$.

(2) (Convexity) If $c, c^{\prime}:[0,1] \rightarrow X$ are constant speed parametrisations of geodesic segments, then, for all $t \in[0,1]$,

$$
d\left(c(t), c^{\prime}(t)\right) \leq(1-t) d\left(c(0), c^{\prime}(0)\right)+t d\left(c(1), c^{\prime}(1)\right) .
$$

(3) $X$ is contractible.

Proof. Fact (1) is immediate from the definition of the $\operatorname{CAT}(0)$ condition. If in (2) one assumes that $c$ and $c^{\prime}$ have the same initial point then the asserted convexity property for $X$ follows immediately from the corresponding property of lines in the Euclidean plane, by $\mathrm{CAT}(0)$. Given arbitrary $c$ and $c^{\prime}$ one joins the initial point of $c^{\prime}$ to the terminal point of $c$ by a geodesic segment $\gamma$ and then applies the preceding special case to the pairs $c^{\prime}, \gamma$ and $c, \gamma^{*}$, where the star denotes reversed orientation.

From (1) and (2) it follows that the homotopy, parameterised in $[0,1]$, which pulls each point of $X$ in to a fixed basepoint at constant speed is a Lipschitz contraction of $X$.

Remark. Geodesic metric spaces which satisfy the condition stated in Lemma 1.1(2) are often called convex spaces, and have been studied by Busemann [Bu] and others. In general, such spaces do not satisfy the CAT(0) inequality (see $[\mathrm{BH}]$ ), and Theorem $\mathrm{A}$ is not valid in this more general context. However, Bowditch [Bo] has shown that a slight variation on Theorem A does hold for an arbitrary convex space. 
Notation. Let $X$ be a $\operatorname{CAT}(0)$ space. We shall use the symbol $[x, y]$ to denote the unique geodesic segment from $x$ to $y$ in $X$. We use the symbol $\Delta(x, y, z)$ to denote the unique geodesic triangle in $X$ with vertices $x, y, z$ and $\bar{\Delta}(x, y, z)$ to denote the corresponding comparison triangle.

The following definition of angle in a $\mathrm{CAT}(0)$ space agrees with Alexandrov's notion of supangle [A], and a moment's thought (focusing on the cosine rule) will convince the reader that it agrees with the usual notion of angle in Euclidean space or, indeed, any Riemannian manifold.

Definition of angle. Let $c:[0, a] \rightarrow X$ and $c^{\prime}:\left[0, a^{\prime}\right] \rightarrow X$ be two geodesic segments with $p=c(0)=c^{\prime}(0)$. The angle between $c$ and $c^{\prime}$ at $p$ is defined to be $\lim _{T \rightarrow 0^{+}} A_{c, c^{\prime}}\left(t, t^{\prime}\right)$, where $T=\max \left\{t, t^{\prime}\right\}$, with $t, t^{\prime}>0$, and

$$
A_{c, c^{\prime}}\left(t, t^{\prime}\right):=\arccos \frac{1}{2 t t^{\prime}}\left(t^{2}+t^{\prime 2}-d\left(c(t), c^{\prime}\left(t^{\prime}\right)\right)^{2}\right) \text {. }
$$

Notice that this definition depends only on the germs of $c(0)$ and $c^{\prime}(0)$ at $p$. It is well defined because of Lemma 1.2 , which is an easy consequence of the $\mathrm{CAT}(0)$ inequality.

The angle at the vertex of a geodesic triangle in a $\operatorname{CAT}(0)$ space is defined to be the angle between those sides of $\Delta$ which are incident at that vertex. We adopt the notation $x \hat{p} y$ for the angle between $[p, x]$ and $[p, y]$ at $p$.

Lemma 1.2. $A_{c, c^{\prime}}\left(t, t^{\prime}\right)$ is a continuous non-decreasing function of both $t, t^{\prime}>$ 0 . (In the case where $X$ is Euclidean space this function is constant.)

Definition. Let $Y$ be a metric space. A function $f: Y \rightarrow \mathbb{R}$ is said to be upper semicontinuous if $f(y) \geq \lim \sup _{n \rightarrow \infty} f\left(y_{n}\right)$ whenever $y_{n} \rightarrow y$.

An upper semicontinuous function attains its supremum on a compact set but does not necessarily attain its infimum.

In subsequent sections we shall make extensive use of the following observations concerning angles in $\mathrm{CAT}(0)$ spaces.

Lemma 1.3. Let $X$ be a $\mathrm{CAT}(0)$ space.

(1) $x \hat{p} z \leq x \hat{p} y+y \hat{p} z$ for all $x, y, z, p \in X$.

(2) For every geodesic triangle $\Delta$ in $X$ the angle at each vertex of $\Delta$ is no greater than the corresponding angle in the comparison triangle $\bar{\Delta}$.

(3) For fixed $p$, the angle $x \hat{p} y$ is a continuous function of $x$ and $y$.

(4) For fixed $x$ and $y$, the angle $x \hat{p} y$ is an upper semicontinuous function of $p$.

(5) $[x, y]$ is the concatenation of $[x, p]$ and $[p, y]$ if and only if the angle between $[p, x]$ and $[p, y]$ at $p$ is $\pi$.

Proof. (1) and (2) are immediate from the definition of angle and the CAT(0) condition. (3) is a corollary of Lemma 1.1(2). For (4), consider $p_{n} \rightarrow p$. Given $\epsilon>0$ fix $T>0$ such that $x \widehat{p} y>A_{[p, x],[p, y]}(t, t)-\epsilon$ for all $t \leq T$. By Lemma 1.1(2), for sufficiently large $n$ we have that $A_{\left[p_{n}, x\right],\left[p_{n}, y\right]}(T, T)<$ $A_{[p, x],[p, y]}(T, T)+\epsilon$. Since $A_{\left[p_{n}, x\right],\left[p_{n}, y\right]}(T, T) \geq x \widehat{p}_{n} y$ and $\epsilon>0$ is arbitrary, this establishes (4). 
The "only if" implication of (5) is clear from the definition of $A_{c, c^{\prime}}\left(t, t^{\prime}\right)$. The converse follows from (2) and the observation that $[x, y]$ is the concatenation of $[x, p]$ and $[p, y]$ if and only if the comparison triangle with vertices $\bar{x}, \bar{p}, \bar{y}$ is degenerate and $\bar{p} \in[\bar{x}, \bar{y}]$.

Proposition 1.4. Let $X$ be a $\mathrm{CAT}(0)$ space, and let $\Delta$ be a geodesic triangle in $X$. If one of the vertex angles of $\Delta$ is equal to the corresponding angle in the comparison triangle $\bar{\Delta}$, then the natural map $\bar{\Delta} \rightarrow \Delta$, which sends each comparison point $\bar{x}$ to $x$, can be extended to an isometric embedding of the convex hull of $\bar{\Delta}$ into $X$.

Proof. Consider $\Delta=\Delta\left(x_{1}, x_{2}, x_{3}\right)$, with comparison triangle $\Delta\left(\bar{x}_{1}, \bar{x}_{2}, \bar{x}_{3}\right)=$ $\bar{\Delta}\left(x_{1}, x_{2}, x_{3}\right) \subseteq \mathbb{E}^{2}$, and suppose that $x_{1} \hat{x}_{2} x_{3}=\bar{x}_{1} \hat{\bar{x}}_{2} \bar{x}_{3}$. The first stage of the proof is to show that $d(\bar{y}, \bar{z})=d(y, z)$ for all points $y, z$ on the sides of $\Delta$. If $d(\bar{y}, \bar{z})>d(y, z)$, then, from Lemma 1.2 and the definition of angle, it follows that the angle between the sides of $\bar{\Delta}\left(x_{1}, x_{2}, x_{3}\right)$ containing $\bar{y}$ and $\bar{z}$ is strictly greater than the corresponding angle in $\Delta$. Thus we can reduce to the case where $z \in\left[x_{1}, x_{3}\right]$. Henceforth we assume that this is the case.

We claim that $d\left(x_{2}, z\right)=d\left(\bar{x}_{2}, \bar{z}\right)$. For if this were not the case, then $d\left(x_{2}, z\right)<d\left(\bar{x}_{2}, \bar{z}\right)$, and the cosine rule would then imply that the sum of the angles $\bar{x}_{1} \hat{\bar{x}}_{2} \bar{z}$ and $\bar{z} \widehat{\bar{x}}_{2} \bar{x}_{3}$ was strictly greater than the sum of the corresponding angles in the comparison triangles $\bar{\Delta}\left(x_{1}, x_{2}, z\right)$ and $\bar{\Delta}\left(z, x_{2}, x_{3}\right)$. But these latter angles are at least as large as $x_{1} \widehat{x}_{2} z$ and $z \widehat{x}_{2} x_{3}$ respectively (Lemma 1.3(2)), and hence (Lemma 1.3(1)) we would have that

$$
\bar{x}_{1} \widehat{\bar{x}}_{2} \bar{z}+\bar{z} \widehat{\bar{x}}_{2} \bar{x}_{3}>x_{1} \widehat{x}_{2} x_{3}=\bar{x}_{1} \widehat{\bar{x}}_{2} \bar{x}_{3} \text {, }
$$

which is nonsense.

The preceding argument shows that $\Delta\left(\bar{x}_{1}, \bar{x}_{2}, \bar{z}\right)$ and $\Delta\left(\bar{z}, \bar{x}_{2}, \bar{x}_{3}\right)$ are comparison triangles for $\Delta\left(x_{1}, x_{2}, z\right)$ and $\Delta\left(z, x_{2}, x_{3}\right)$ respectively. In particular, $\bar{x}_{1} \widehat{\bar{z}} \bar{x}_{2} \geq x_{1} \widehat{z} x_{2}$ and $\bar{x}_{1} \widehat{\bar{z}} \bar{x}_{3} \geq x_{1} \widehat{z} x_{3}$, by Lemma 1.3(2). In fact we must have equality in both of these relations, because $x_{1} \widehat{z} x_{2}+x_{1} \widehat{z} x_{3} \geq \pi$, by Lemma $1.3(5)$. Thus we can apply the argument of the preceding paragraph to each of the triangles $\Delta\left(\bar{x}_{1}, \bar{x}_{2}, \bar{z}\right)$ and $\Delta\left(\bar{z}, \bar{x}_{2}, \bar{x}_{3}\right)$, casting $z$ in the role previously played by $x_{2}$. Thus we see that $d(\bar{y}, \bar{z})=d(y, z)$, and deduce that the map $\bar{\Delta} \rightarrow \Delta$, which sends each comparison point $\bar{x}$ to $x$, is an isometry.

The desired extension of this map to the convex hull of $\bar{\Delta}$ is defined by sending each of the line segments $\left[\bar{x}_{2}, \bar{z}\right]$, for $\bar{z} \in\left[\bar{x}_{1}, \bar{x}_{3}\right]$, onto $\left[x_{2}, z\right]$ by an isometry. The first stage of the proof shows that, given any $\bar{z}, \bar{z}^{\prime} \in\left[\bar{x}_{1}, \bar{x}_{3}\right]$, the triangle $\Delta\left(\bar{z}, \bar{x}_{2}, \bar{z}^{\prime}\right)$ is a comparison triangle for $\Delta\left(z, x_{2}, z^{\prime}\right)$. Hence, by CAT $(0)$, the map from the convex hull of $\Delta\left(\bar{x}_{1}, \bar{x}_{2}, \bar{x}_{3}\right)$ into $X$ is (weakly) contracting. But every geodesic segment in the convex hull can be extended to a geodesic segment meeting the boundary, and the map restricts to an isometry on the boundary. Hence, by the triangle inequality, the extended map must be an isometry onto its image.

\section{Geodesic rays.}

Definition. Let $X$ be a geodesic metric space. A geodesic ray in $X$ is an isometric embedding $c:[0, \infty) \rightarrow X$. A geodesic is an isometric embedding of the real line. Two geodesic rays (respectively geodesics) $c, c^{\prime}$ are said to be asymptotic if there exists a constant $D$ such that $d\left(c(t), c^{\prime}(t)\right)<D$ for all $t$. 
Recall that a metric space is said to be proper if all of its closed bounded subsets are compact.

Lemma 1.5. Let $X$ be a proper $\mathrm{CAT}(0)$ space. For every $p \in X$ and every geodesic ray $c:[0, \infty) \rightarrow X$ there exists a unique geodesic ray $c^{\prime}:[0, \infty) \rightarrow X$ asymptotic to $c$ for which $c^{\prime}(0)=p$.

Proof. The uniqueness assertion is clear from the convexity of the metric on $X$ (Lemma 1.1(2)). In order to establish the existence of $c^{\prime}$ one considers the sequence of geodesic segments $\gamma_{n}:=[p, c(n)]$. Using the fact that the closed ball of radius $r$ centered at $p$ is compact, one proceeds by recursion on $r \in \mathbb{N}$ to extract a subsequence of integers $\left\{n_{r}(i)\right\}_{i}$ from $\left\{n_{r-1}(i)\right\}_{i}$ such that the sequence $\left\{\gamma_{n_{r}(i)}(k)\right\}_{i}$ converges in $X$ for all integers $k \leq r$. The diagonal sequence $m(r):=n_{r}(r)$ yields a sequence of geodesic segments $\gamma_{m(r)}$ whose pointwise limit is the desired geodesic ray $c^{\prime}$.

Definition/Notation. For pairs of geodesic rays in a $\operatorname{CAT}(0)$ space $X$, being asymptotic is clearly an equivalence relation; we denote the set of equivalence classes by $\mathscr{G}_{X}$. Henceforth we shall denote elements of $\mathscr{G}_{X}$ by $c$ (or $c^{\prime}, \tilde{c}$, etc.), and when it is necessary to consider a sequence of elements from $\mathscr{G}_{X}$ we shall use upper indices: $c^{(n)}$.

Given $p \in X$ and $c \in \mathscr{G}_{X}$ we shall use the symbol $c_{p}$ to denote the unique element of the class $c$ such that $c_{p}(0)=p$. We shall use the notation $\left\langle c, c^{\prime}\right\rangle_{p}$ for the angle between $c_{p}$ and $c_{p}^{\prime}$ at $p$.

We topologize $\mathscr{G}_{X}$ by decreeing that a subset $K \subseteq \mathscr{G}_{X}$ is closed if and only if $c \in K$ whenever there exists $p \in X$ and $c^{(n)} \in K$ such that $c_{p}^{(n)} \rightarrow c_{p}$ pointwise (in which case we write $c^{(n)} \rightarrow c$ ); the following lemma shows that this definition is independent of the choice of $p$. Lemma 1.1(2) shows that pointwise convergence for geodesic rays in a $\mathrm{CAT}(0)$ space is the same as uniform convergence on compact subsets.

Lemma 1.6. Let $X$ be a proper $\mathrm{CAT}(0)$ space and consider $c \in \mathscr{G}_{X}$. Then $c^{(n)} \rightarrow c$ if and only if $c_{q}^{(n)} \rightarrow c_{q}$ for all $q \in X$.

Proof. For all $q, q^{\prime} \in X$ and all $c^{\prime} \in \mathscr{G}_{X}$ the function $t \mapsto d\left(c_{q}^{\prime}(t), c_{q^{\prime}}^{\prime}(t)\right)$ is convex and bounded and, hence, non-increasing. Thus, for any $p, q \in X$ one knows that $d\left(c_{p}^{(n)}(t), c_{q}^{(n)}(t)\right) \leq d(p, q)$ and, similarly, $d\left(c_{p}(t), c_{q}(t)\right) \leq$ $d(p, q)$. In light of this observation, the lemma follows easily from the cosine rule in plane geometry:

Given $K>0, t>0$, and $\epsilon>0$, the cosine rule yields a number $L=L(\epsilon, t)$ such that if two geodesic segments $\ell_{1}$ and $\ell_{2}$ in $\mathbb{E}^{2}$ with $\ell_{1}(0)=\ell_{2}(0)$ have terminal endpoints that are a distance at most $K$ apart and both have length at least $L$, then $d\left(\ell_{1}(t), \ell_{2}(t)\right) \leq \epsilon$. It follows easily from the $\operatorname{CAT}(0)$ condition that, under the same hypotheses on $\ell_{1}$ and $\ell_{2}$, this last inequality holds in any $\mathrm{CAT}(0)$ space.

We shall make liberal use of the following compactness properties.

Lemma 1.7. Let $X$ be a proper geodesic metric space.

(1) Consider a sequence of geodesic segments $\gamma_{n}:\left[0, T_{n}\right] \rightarrow X$ with common initial point $p$. If $T_{n} \rightarrow \infty$ as $n \rightarrow \infty$, then there is a subsequence $\gamma_{n(i)}$ and $c \in \mathscr{G}_{X}$ such that the paths $\gamma_{n(i)}$ converge to $c_{p}$ pointwise.

(2) Every sequence $c^{(n)}$ in $\mathscr{G}_{X}$ contains a convergent subsequence. 
Proof. The proof of (1) is entirely similar to Lemma 1.5. By fixing $p$ we can reduce (2) to a special case of (1), with $\gamma_{n}:=\left.c_{p}^{(n)}\right|_{[0, n]}$.

Lemma 1.8. Let $X$ be a proper $\mathrm{CAT}(0)$ space.

(1) Fix $p \in X$, and let $\left\{x_{n}\right\},\left\{y_{n}\right\}$ be sequences of points in $X$. Suppose that $c, c^{\prime} \in \mathscr{G}_{X}$ are such that $\left[p, x_{n}\right] \rightarrow c$ and $\left[p, y_{n}\right] \rightarrow c^{\prime}$, pointwise, as $n \rightarrow \infty$. Then $x_{n} \widehat{p} y_{n} \rightarrow\left\langle c, c^{\prime}\right\rangle_{p}$ as $n \rightarrow \infty$.

(2) If $c^{(n)} \rightarrow c$ and $\tilde{c}^{(n)} \rightarrow \tilde{c}$ are convergent sequences in $\mathscr{G}_{X}$, then $\left\langle c^{(n)}, \tilde{c}^{(n)}\right\rangle_{p}$ $\rightarrow\langle c, \tilde{c}\rangle_{p}$ for all $p \in X$.

(3) The angle $\left\langle c, c^{\prime}\right\rangle_{p}$ is an upper semicontinuous function of $p$. Indeed, for every $p \in X$ and $\epsilon>0$ there exists $\delta>0$ such that $\left\langle c, c^{\prime}\right\rangle_{q}-\left\langle c, c^{\prime}\right\rangle_{p}<$ $\epsilon$ for all $c, c^{\prime} \in \mathscr{G}_{X}$ whenever $d(p, q)<\delta$.

Proof. As with the corresponding assertion in Lemma 1.3, part (1) of Lemma 1.8 follows easily from the convexity properties of the metric in a $\operatorname{CAT}(0)$ space. Part (2) is a direct consequence of (1) and Lemma 1.6. For (3), given $p \in X$ and $\epsilon>0$, we use (2) and the compactness of $\mathscr{G}_{X}$ to find $T>0$ such that $A_{c_{p}, c_{p}^{\prime}}(t, t)-\left\langle c, c^{\prime}\right\rangle_{p}<\epsilon / 2$ for all $c, c^{\prime} \in \mathscr{G}_{X}$ and all $t \leq T$. We then fix $0<\eta<1$ such that if $\alpha, \beta \in[0, \pi]$ satisfy $|\cos \alpha-\cos \beta|<3 \eta$, then $|\alpha-\beta|<\epsilon / 2$. Let $\delta=\eta T$. If $d(p, q)<\delta$, then an easy estimate using the definition of angle yields $\left|\cos A_{c_{p}, c_{p}^{\prime}}(T, T)-\cos A_{c_{q}, c_{q}^{\prime}}(T, T)\right|<3 \eta$ for all $c, c^{\prime} \in \mathscr{G}_{X}$. Hence $\left\langle c, c^{\prime}\right\rangle_{q}-\left\langle c, c^{\prime}\right\rangle_{p}<A_{c_{q}, c_{q}^{\prime}}(T, T)-\left[A_{c_{p}, c_{p}^{\prime}}(T, T)-\epsilon / 2\right]<\epsilon$.

Remark. One can view $\mathscr{G}_{X}$ as the ideal points of the natural compactification of $X$ obtained by taking the projective limit of the system of closed balls about a fixed base point, where the binding maps in the inverse system are given by orthogonal projection (see [BGS], $[\mathrm{BH}]$ ). For the sake of brevity, we shall not expound upon this point in this article, but it forms the natural backdrop for a number of our arguments. For example, Lemma 1.3(3) and Lemma 1.8(1), (2) can be combined into the statement that $x \widehat{p} y$ is a continuous function of $x$ and $y$ as these points vary over the compactification of $X$.

The following observation will be needed in the next section.

Lemma 1.9. Let $X$ be a proper CAT(0) space. For every pair of distinct classes $c, c^{\prime} \in \mathscr{G}_{X}$ there exists $p \in X$ such that $\left\langle c, c^{\prime}\right\rangle_{p}>0$.

Proof. Let $p \in X$ be arbitrary, and fix $t$ sufficiently large to ensure that $d\left(c_{p}(t), c_{p}^{\prime}(t)\right)>0$. If the angle $\left\langle c, c^{\prime}\right\rangle_{c_{p}(t)}$ were zero, then, by Lemma 1.3(5), the angle between $\left[c_{p}(t), p\right]$ and $c_{c_{p}(t)}^{\prime}$ at $c_{p}(t)$ would be $\pi$. Hence (again by Lemma 1.3(5)) the concatenation of $\left[p, c_{p}(t)\right]$ and $c_{c_{p}(t)}$ would be a geodesic ray, contradicting the fact that $c_{p}$ is the only geodesic ray from $p$ that is asymptotic to $c^{\prime}$.

\section{VISIBILITY AND HYPERBOLICITY}

The notion of visibility for simply connected manifolds of non-positive curvature was introduced by Eberlein and O'Neill [EO] as a generalisation of strictly negative curvature. This condition can be interpreted in a number of different ways (see [BGS, p. 54]), most of which can be generalised to CAT(0) spaces (see $[\mathrm{BH}])$. The proof of our main result relies on the equivalence of three of these 
definitions in order to bridge the gap between visibility spaces and $\delta$-hyperbolic spaces. The following definition of visibility is what Eberlein and O'Neill [EO] call Axiom 1.

Definition. A CAT(0) space $X$ is said to be a visibility space if for every pair of non-asymptotic geodesic rays $c, c^{\prime}:[0, \infty) \rightarrow X$ there exists a constant $D>0$ and a geodesic $\gamma: \mathbb{R} \rightarrow X$ such that $d(\gamma(t), c(t)) \leq D$ and $d\left(c^{\prime}(t), \gamma(-t)\right) \leq D$ for all $t>0$. In other words, $\gamma$ is asymptotic to $c$ in the forward direction and asymptotic to $c^{\prime}$ in the backward direction.

Definition. A CAT(0) space $X$ is said to be locally visible if for every $p \in X$ and $\epsilon>0$ there exists $R(p, \epsilon) \geq 0$ such that if a geodesic segment $[x, y]$ lies entirely outside the ball of radius $R(p, \epsilon)$ about $p$, then $x \widehat{p} y<\epsilon$.

Definition. A CAT(0) space $X$ is said to be uniformly visible if for every $\epsilon>0$ there exists $R(\epsilon) \geq 0$ such that, given $p \in X$, if a geodesic segment $[x, y]$ lies entirely outside the ball of radius $R(\epsilon)$ about $p$, then $x \widehat{p} y<\epsilon$.

Definition. Given $\delta>0$, a geodesic metric space $X$ is said to be $\delta$-hyperbolic if for every geodesic triangle $\Delta \subseteq X$ each edge of $\Delta$ is contained in the $\delta$-neighbourhood of the union of the other two sides. $X$ is said to be hyperbolic (in the sense of Gromov) if it is $\delta$-hyperbolic for some $\delta$.

Proposition 2.1. Let $X$ be a proper CAT(0) space.

(1)

$$
\begin{aligned}
X \text { is hyperbolic } & \Longleftrightarrow X \text { is uniformly visible } \\
& \Longleftrightarrow X \text { is locally visible } \\
& \Longleftrightarrow X \text { is a visibility space } .
\end{aligned}
$$

(2) If $X$ is cocompact and locally visible then it is uniformly visible (hence hyperbolic).

Remark. There exist non-proper CAT(0) spaces which are locally visible but not visiblity spaces and vice versa. An example of a visibility space which is not locally visible can be obtained by taking any visibility space, fixing a basepoint and attaching to that point the corner of a Euclidean square of side $n$ for every positive integer $n$. An example of a locally visible space which is not visible is $\left\{(x, y) \mid 1>y \geq 1-(1+|x|)^{-1}\right\}$ with the path metric induced from the Euclidean plane. Examples of $\operatorname{CAT}(0)$ visibility spaces which not uniformly visible can be obtained by considering negatively curved 1-connected manifolds whose curvature is not bounded above by a negative constant.

Proof of Proposition 2.1. We first prove (2). Suppose that $X$ is cocompact, covered by the translates of compact $K$, say, and locally visible. Numbers $R(p, \epsilon)$ as required by the definition of local visibility can then be defined by

$$
R(p, \epsilon):=\inf \left\{\rho \mid \forall x, y \in X \text { with } x \widehat{p} y>\epsilon,[x, y] \text { intersects } \operatorname{Ball}_{\rho}(p)\right\} .
$$

Isometries preserve angles, so for all $p \in X$ there exists $p^{\prime} \in K$ with $R(p, \epsilon)=$ $R\left(p^{\prime}, \epsilon\right)$. Furthermore, for fixed $x$ and $y$ the angle $x \hat{p} y$ is an upper semicontinuous function of $p$, so $R(p, \epsilon)$ is also an upper semicontinuous function of $p$ and, hence, is bounded on the compact set $K$. 
We begin the proof of (1) by supposing that $X$ is $\delta$-hyperbolic. Consider a geodesic triangle in $X$ with vertices $p, x, y$, and suppose that the distance from $p$ to $[x, y]$ is greater than $\rho \geq 2 \delta$. Because $X$ is $\delta$-hyperbolic, we can find $z$ on $[x, y], u$ on $[p, x]$, and $v$ on $[p, y]$ such that $d(z, u), d(z, v) \leq$ $\delta$. Thus $d(u, v) \leq 2 \delta$ and $d(p, u), d(p, v) \geq \rho-\delta$. But, if $\theta=u \widehat{p} v$, then applying Lemma 1.3(2) to the comparison triangle with vertices $\bar{p}, \bar{u}, \bar{v}$ we see that $d(u, v) \geq(\rho-\delta) \sin \theta$. Thus

$$
\theta \leq \arcsin [2 \delta /(\rho-\delta)] .
$$

This bound is independent of $p$; thus, $X$ is uniformly visible.

Conversely, if $X$ is uniformly visible, then $X$ is $\delta$-hyperbolic with $\delta=$ $R(\pi / 2)$. To see this, consider a geodesic triangle with vertices $x, y, z$ and a point $p$ on the side $[x, y]$. The angles $x \widehat{p} z$ and $y \widehat{p} z$ must sum to at least $\pi$ (Lemma 1.3(1 and 5)), so one of these angles, $x \widehat{p} z$ say, must be at least $\pi / 2$. Thus, by definition of uniform visibility, $d(p,[x, z]) \leq R(\pi / 2)$.

Next we show that a locally visible space is a visibility space. Fix distinct equivalence classes of rays $c, c^{\prime} \in \mathscr{G}_{X}$ and $p \in X$ such that $\left\langle c, c^{\prime}\right\rangle_{p}=\epsilon>0$ (Lemma 1.9). If $X$ is locally visible, then for every $n \in \mathbb{N}$ the geodesic segment [ $\left.c_{p}(n), c_{p}^{\prime}(n)\right]$ must enter the ball of radius $R(p, \epsilon)$ about $p$. Thus we obtain a sequence of points $q_{n}$ with $q_{n} \in\left[c_{p}(n), c_{p}^{\prime}(n)\right]$ and $d\left(p, q_{n}\right)<R(p, \epsilon)$, and using the fact that $X$ is proper we can extract a convergent subsequence, with limit $q$ say. We claim that the concatenation of $c_{q}$ and $c_{q}^{\prime}$, reversing the orientation on the latter, yields the desired geodesic with ends $c$ and $c^{\prime}$. For if this were not the case, then for some $\eta>0$ and some points $x$ on $c_{q}$ and $y$ on $c_{q}^{\prime}$ we would have $d(x, y)<d(x, q)+d(y, q)-\eta$. But since the sequences of geodesic segments $\left[q, c_{p}(n)\right]$ and $\left[q, c_{p}^{\prime}(n)\right]$ converge to $c_{q}$ and $c_{q}^{\prime}$ pointwise, this would imply that for all sufficently large $n$ there would exist $x_{n}$ on $\left[q, c_{p}(n)\right]$ and $y_{n}$ on $\left[q, c_{p}^{\prime}(n)\right]$ such that $d\left(x_{n}, y_{n}\right)<d\left(x_{n}, q\right)+$ $d\left(y_{n}, q\right)-\eta$. On the other hand, $d\left(x_{n}, q_{n}\right)+d\left(q_{n}, y_{n}\right)=d\left(x_{n}, y_{n}\right)$. Thus we would obtain a contradiction to the fact that $q$ was defined as a limit point of the $q_{n}$.

What remains to be proved is that a proper visibility space is locally visible. We shall actually prove something stronger. Namely, we shall show that if $X$ is not locally visible, then there exist $c, c^{\prime} \in \mathscr{G}_{X}$ such that $\left\langle c, c^{\prime}\right\rangle_{p^{\prime}} \leq \pi / 2$ for all $p^{\prime} \in X$. The following argument is due to P. Eberlein [E].

If $X$ is not locally visible, then there exist $p \in X, \epsilon>0$, and a geodesic segment $\left[x_{n}, y_{n}\right]$ for every $n>0$ such that the angles $x_{n} \widehat{p} y_{n}$ are bounded below by $\epsilon$ and the distance from $p$ to $\left[x_{n}, y_{n}\right]$ is at least $n$. Let $q_{n}$ be the unique point of $\left[x_{n}, y_{n}\right]$ that is closest to $p$. Such a closest point exists because $\left[x_{n}, y_{n}\right]$ is compact, and it is unique because if $q_{n}^{\prime}$ were a second such point, then the comparison triangle with vertices $\bar{p}, \bar{q}_{n}, \bar{q}_{n}^{\prime}$ would be isosceles and hence the angles at $\bar{q}_{n}$ and $\bar{q}_{n}^{\prime}$ would be acute. But the $\operatorname{CAT}(0)$ condition would then yield a point on $\left[\bar{q}_{n}, \bar{q}_{n}^{\prime}\right]$ (and hence $\left[x_{n}, y_{n}\right]$ ) that would be closer to $p$ than the supposedly closest point $q_{n}$. By similar reasoning, the minimality of $d\left(p, q_{n}\right)$ also implies that $p \widehat{q}_{n} x_{n} \geq \pi / 2$ if $x_{n} \neq q_{n}$.

By reversing the roles of $x_{n}$ and $y_{n}$ if necessary and appealing to Lemma 1.3(1), we may pass to a subsequence such that $q_{n} \widehat{p} x_{n}>\epsilon / 2$ for all $n$. By Lemma 1.7(1), we can extract a subsequence of integers $m$ such that the sequences of geodesic segments $\left[p, x_{m}\right]$ and $\left[p, q_{m}\right]$ converge pointwise to 
geodesic rays based at $p$. We denote these rays by $c_{p}$ and $c_{p}^{\prime}$ respectively. The angle between these rays at $p$ is bounded below by $\epsilon / 2$, so in particular they are distinct. We claim that there is no geodesic in $X$ which is asymptotic to $c$ and $c^{\prime}$ in its forward and backward directions respectively. It is enough to show that $\left\langle c, c^{\prime}\right\rangle_{p^{\prime}} \leq \pi / 2$ for all $p^{\prime} \in X$. It is clear from the cosine rule in Euclidean geometry, together with Lemma $1.3(2)$, that $p \widehat{q}_{m} p^{\prime} \rightarrow 0$ as $m \rightarrow \infty$. But $p \widehat{q}_{m} x_{m} \geq \pi / 2$ so, by Lemma 1.3(1), for all $\delta>0$ and all fixed $p^{\prime}$ the angle $p^{\prime} \widehat{q}_{m} x_{m}$ is at least $\pi / 2-\delta$ for large $m$. The sum of the vertex angles in the geodesic triangle with vertices $p^{\prime}, q_{m}, x_{m}$ is at most $\pi$ (Lemma 1.3(2)), and hence $x_{m} \widehat{p}^{\prime} q_{m}$ is bounded above by $\pi / 2+\delta$ for large $m$. But $\delta$ was arbitrary, and as $m \rightarrow \infty,\left[p^{\prime}, x_{m}\right] \rightarrow c_{p^{\prime}}$ and $\left[p^{\prime}, q_{m}\right] \rightarrow c_{p^{\prime}}^{\prime}$ pointwise. Hence, by Lemma $1.8(1)$, we have that $\left\langle c, c^{\prime}\right\rangle_{p^{\prime}} \leq \pi / 2$.

\section{THE PROOF OF THEOREM A}

In light of Proposition 2.1, the proof of Theorem A will be complete if we show that any cocompact $\mathrm{CAT}(0)$ space $X$ which is not uniformly visible contains a flat plane. The following general result about metric spaces reduces this problem to showing that $X$ contains arbitrarily large flat discs.

Lemma 3.1. Let $Y$ be a separable metric space with basepoint $y_{0}$, and let $X$ be a proper cocompact metric space. If for all $n \in \mathbb{N}$ there exists an isometric embedding $\phi_{n}: \operatorname{Ball}_{n}\left(y_{o}\right) \hookrightarrow X$, then there exists an isometric embedding $\phi$ : $Y \hookrightarrow X$.

Proof. Let $y_{0}, y_{1}, \ldots$ be a countable dense set in $Y$. Because $X$ is cocompact, we can replace $\phi_{n}$ by a suitable choice of $g_{n} \phi_{n}$ with $g_{n} \in \operatorname{Isom}(X)$ and hence assume that the sequence $\left\{\phi_{n}\left(y_{o}\right)\right\}_{n}$ is contained in a compact (hence bounded) subset of $X$. Since $X$ is proper, it follows that for all $i$ the sequence $\left\{\phi_{n}\left(y_{i}\right)\right\}_{n}$ is contained in a compact subset of $X$. Hence there exists $x_{0} \in X$ and a sequence of integers $\left\{n_{0}(j)\right\}_{j}$ such that $\phi_{n_{0}(j)}\left(y_{0}\right) \rightarrow x_{0}$ as $j \rightarrow \infty$. And proceeding by recursion on $r$, we can find a sequence of elements $x_{n} \in X$ and sequences of integers $\left\{n_{r}(j)\right\}_{j} \subset\left\{n_{r-1}(j)\right\}_{j}$ such that $\phi_{n_{r}(j)}\left(y_{k}\right) \rightarrow x_{k}$ as $j \rightarrow \infty$ for all $k \leq r$. The diagonal sequence $m=n_{r}(r)$ satisfies $\phi_{m}\left(y_{k}\right) \rightarrow x_{k}$, as $m \rightarrow \infty$, for all $k \geq 0$. Since the $\phi_{m}$ are isometries, so too is the map $y_{k} \mapsto x_{k}$. The set $\left\{y_{k}\right\}$ is dense in $Y$, so $y_{k} \mapsto x_{k}$ has a unique extension, which is the desired $\phi$.

The following lemma completes the proof of Theorem A. The idea of the proof is the same as in the Riemannian case (see [BGS], [E]).

Lemma 3.2. Let $X$ be a proper $\mathrm{CAT}(0)$ space. If $X$ is not a visibility space then one can isometrically embed arbitrarily large flat discs into $X$.

Proof. If $X$ is not a visibility space, then, as in the proof of Proposition 2.1, there exist distinct classes of geodesic rays $c, c^{\prime} \in \mathscr{G}_{X}$ such that $\left\langle c, c^{\prime}\right\rangle_{p} \leq \pi / 2$ for all $p \in X$. Hence

$$
\left\langle\phi(c), \phi\left(c^{\prime}\right)\right\rangle_{\phi(p)}=\left\langle c, c^{\prime}\right\rangle_{p} \leq \pi / 2
$$

for all $p \in X$ and all $\phi \in \operatorname{Isom}(X)$. Let

$$
\theta(p):=\sup \left\{\left\langle\phi(c), \phi\left(c^{\prime}\right)\right\rangle_{p} \mid \phi \in \operatorname{Isom}(X)\right\} .
$$


Notice that $\theta(p) \leq \pi / 2$ and that $\theta(p)$ is invariant under the action of $\operatorname{Isom}(X)$. Furthermore, by Lemma 1.8(3), $\theta(p)$ is upper semicontinuous. Thus, since we are assuming that $X$ is cocompact, $\theta(p)$ attains its supremum at some point $p_{0} \in X$. Finally, by Lemma 1.7(2), we can choose a sequence $\left\{\phi_{n}\right\}_{n} \subset \operatorname{Isom}(X)$ such that $\left\langle\phi_{n}(c), \phi_{n}\left(c^{\prime}\right)\right\rangle_{p_{0}} \rightarrow \theta\left(p_{0}\right)$ and both of the sequences $\left\{\phi_{n}(c)\right\}_{n}$ and $\left\{\phi_{n}\left(c^{\prime}\right)\right\}_{n}$ converge in $\mathscr{G}_{X}$. For convenience we shall again denote the limit rays by $c$ and $c^{\prime}$ respectively. By Lemma 1.8(2) we have that

$$
\left\langle c, c^{\prime}\right\rangle_{p_{0}}=\theta\left(p_{0}\right) \text {. }
$$

Given $t>0$, let $p_{t}$ denote the point $c_{p_{0}}(t)$. We shall prove that the geodesic triangle whose vertices are $p_{0}, p_{t}$ and $q_{t}:=c_{p_{t}}^{\prime}(t)$ bounds a flat triangular disc in $X$. In order to do so, we also consider the point $r_{t}:=c_{p_{0}}^{\prime}(t)$ and let $\alpha, \beta$, and $\gamma$ denote the angles $p_{t} \widehat{p}_{0} q_{t}, \quad p_{0} \widehat{p}_{t} q_{t}$, and $r_{t} \widehat{p}_{0} q_{t}$ respectively. By definition, $d\left(p_{0}, p_{t}\right)=d\left(p_{t}, q_{t}\right)=d\left(p_{0}, r_{t}\right)=t$, and because $c_{p_{0}}^{\prime}$ and $c_{p_{t}}^{\prime}$ are asymptotic, $s \mapsto d\left(c_{p_{0}}^{\prime}(s), c_{p_{t}}^{\prime}(s)\right)$ is convex and bounded, hence non-increasing, so $d\left(r_{t}, q_{t}\right) \leq t$.

Consider the Euclidean quadrilateral which one obtains by joining comparison triangles $\bar{\Delta}\left(p_{0}, p_{t}, q_{t}\right)$ and $\bar{\Delta}\left(p_{0}, q_{t}, r_{t}\right)$ along $\left[\bar{p}_{0}, \bar{q}_{t}\right]$. The above estimates on the lengths of the sides of this quadrilateral mean that the comparison angles must satisfy $\bar{\alpha}+\bar{\beta}+\bar{\gamma} \leq \pi$. But $\bar{\alpha}+\bar{\gamma} \geq \alpha+\gamma \geq \theta\left(p_{0}\right)$, by Lemma 1.3(1), (2). And $\bar{\beta} \geq \beta \geq \pi-\theta\left(p_{t}\right)$, by parts (1), (2), and (5) of Lemma 1.3. Combining all of these inequalities with the fact that $\theta\left(p_{t}\right) \leq \theta\left(p_{0}\right)$ (because $\theta\left(p_{0}\right)$ was chosen to be maximal), we see that

$$
\bar{\beta}=\beta=\pi-\theta\left(p_{0}\right) \text {. }
$$

Hence, by Proposition 1.4, the geodesic triangle whose vertices are $p_{0}, p_{t}$, and $q_{t}$ bounds a flat triangular disc in $X$. Furthermore, we have shown that the angle $\beta$ is independent of $t$, so as $t \rightarrow \infty$ the inscribed radius of this triangular disc tends to infinity.

\section{APPENDIX: SUBQUADRATIC ISOPERIMETRIC INEQUALITIES}

We conclude this article with a remark on isoperimetric inequalities. Since this remark is rather technical and likely only to be of interest to specialists, we give only the briefest review of the definitions involved. In particular, with apologies, it does not seem appropriate to review the geometric interpretation of isoperimetric inequalities in terms of van Kampen diagrams (see [LS]) or basic facts about quasi-isometries [GH].

Recall that, given any positive function $f: \mathbb{N} \rightarrow \mathbb{R}_{+}$, a finitely presented group $\Gamma=\langle\mathscr{A} \mid \mathscr{R}\rangle$ is said to satisfy an isoperimetric inequality of type $f$ if there exists a constant $K$ such that every reduced word $w \in \operatorname{ker}(F(\mathscr{A}) \rightarrow \Gamma)$ of length at most $n$ can be expressed in the free group $F(\mathscr{A})$ as the product of at most $K f(K n+K)+K n+K$ conjugates of relators $r \in \mathscr{R}$. This condition (but not the particular constant $K$ ) is independent of the chosen finite presentation [Al]. Furthermore, the presence of the constant $K$ in this definition ensures that if $f_{1}=O\left(f_{2}\right)$ and $\Gamma$ satisfies an isoperimetric inequality of type $f_{1}$, then $\Gamma$ satifies an isoperimetric inequality of type $f_{2}$. In particular, it makes sense to say that $\Gamma$ satisfies a quadratic, linear, or strictly subquadratic isoperimetric inequality. 
Word-hyperbolic groups can, with effort, be characterised as those finitely presented groups which satisfy a strictly subquadratic isoperimetric inequality; this was pointed out by Gromov in his foundational paper [G], and a detailed proof has been given by Ol'shanski [Ol] (see also [P]). Some geometric intuition into this result can be gleaned from the relatively simple proof which Theorem A affords for the following special case. We recall that every group which acts properly and cocompactly by isometries on a proper CAT $(0)$ space satisfies a quadratic isoperimetric inequality (see, e.g., $[\mathrm{AB}])$. We recall also that it remains an open question as to whether every word-hyperbolic group arises as such a group of isometries.

Proposition 4.1. Let $\Gamma$ be a group which acts properly and cocompactly by isometries on a proper CAT(0) space. Then $\Gamma$ is word-hyperbolic if and only if it satisfies a strictly subquadratic isoperimetric inequality.

Sketch of Proof. It is a straightforward matter to show that word-hyperbolic groups satisfy a linear isoperimetric inequality (see $[\mathrm{GH}],[\mathrm{Sh}]$ ), so we concentrate on the assertion that a discrete cocompact group of isometries $\Gamma$ of a proper CAT(0) space $X$ is word-hyperbolic if it satisfies a strictly subquadratic isoperimetric inequality. Fix a finite presentation $\Gamma=\langle\mathscr{A} \mid \mathscr{R}\rangle$.

By a well-known argument, the given action of $\Gamma$ on $X$ yields a $(\lambda, \epsilon)$ quasi-isometry, $\pi$ say, from $X$ to the Cayley graph of $\Gamma$. If $\Gamma$ is not wordhyperbolic, then $X$ is not hyperbolic (because hyperbolicity is preserved by quasi-isometries between geodesic metric spaces), so Theorem A yields an isometrically embedded flat plane $P \subseteq X$. One establishes the proposition by considering the image under $\pi$ of a square in $P$ of side length $B n$, where $B$ is a constant (to be fixed presently) that depends only on $\lambda, \epsilon$, and the length to the longest relator in $\mathscr{R}$. The map $\pi$ sends the four sides of this square to quasi-geodesics in the Cayley graph of $\Gamma$. These paths can be uniformly approximated by translates of quasi-geodesic words in the generators $\mathscr{A}$, thought of as edge-paths in the Cayley graph in the usual way (see [AB, Section 10]). Let us denote these words by $u_{n}^{1}, u_{n}^{2}, u_{n}^{3}, u_{n}^{4}$. Notice that the length of the concatenation of the $u_{n}^{i}$, which we shall denote $w_{n}$, is bounded above by a linear function of $n$. In order to complete the proof we must obtain a quadratic lower bound on the area of an arbitrary van Kampen diagram for $w_{n}$. Let $p\left(w_{n}\right)$ denote the path which $w_{n}$ determines in the Cayley graph of $\Gamma$.

We choose $B$ so as to ensure that the $u_{n}^{i}$ have length at least $3 n$. Let $v_{n}^{i}$ denote the middle subword of length $n$ in $u_{n}^{i}$. By construction, the subpaths of $p\left(w_{n}\right)$ determined by the words $v_{n}^{i}$ and $v_{n}^{j}(i \neq j)$ are a distance at least $C n$ apart (where $C$ is a constant depending only on the choice of $B$ ). In light of this observation (which is just the translation into $\Gamma$ of the geometry of the flat square with which we started) one can show by an elementary argument in the manner of the appendix to [GS] that the area of the $n$th star neighbourhood of $v_{n}^{1}$ in an arbitrary van Kampen diagram for $w_{n}$ is bounded below by a quadratic function of $n$.

\section{ACKNOWLEDGMENTS}

I would like to thank André Haefliger for numerous stimulating and enjoyable conversations about $\mathrm{CAT}(0)$ spaces. 


\section{REFERENCES}

[A] A.D. Alexandrov, $A$ theorem on triangles in a metric space and some applications, Trudy Mat. Inst. Steklov. 38 (1951), 5-23. (Russian)

[Al] J.M. Alonso, Inégalités isopérimétriques et quasi-isométries, C. R. Acad. Sci. Paris Sér. I Math. 311 (1990), 761-764.

[AB] J.M. Alonso and M.R. Bridson, Semihyperbolic groups, Proc. London Math. Soc. (3) (to appear).

[Ba] W. Ballmann, Singular spaces of non-positive curvature, Sur les Groupes Hyperboliques d'après Mikhael Gromov (E. Ghys and P. de la Harpe, eds.), Progr. Math., vol. 83, Birkhäuser, Boston, MA, 1990.

[BGS] W. Ballmann, M. Gromov, and V. Schroeder, Manifolds of non-positive curvature, Progr. Math., vol. 61, Birkhäuser, Boston, MA, 1990.

[BH] M.R. Bridson and A. Haefliger, Metric spaces of non-positive curvature, in preparation.

[Bo] B.H. Bowditch, Minkowski subspaces of non-positively curved metric spaces, preprint, University of Southampton, 1993.

[Br] M.R. Bridson, Geodesics and curvature in metric simplicial complexes, Group Theory from a Geometric Viewpoint, Proc. ICTP, Trieste (E. Ghys, A. Haefliger, and A. Verjovsky, eds.), World Scientific, Singapore, 1991.

[Bu] H. Busemann, The geometry of geodesics, Academic Press, Reading, MA, 1959.

[CE] J. Cheeger and D.G. Ebin, Comparison theorems in Riemannian geometry, North-Holland, New York, 1975.

[E] P. Eberlein, Geodesic flow in certain manifolds without conjugate points, Trans. Amer. Math. Soc. 167 (1972), 151-170.

[EO] P. Eberlein and B. O’Neill, Visibility manifolds, Pacific J. Math. 46 (1973), 45-110.

[FP] W. Floyd and W. Parry, The growth of nonpositively curved triangles of groups, preprint, VPI \& SU and EMU, 1993.

[GH] E. Ghys ard P. de la Harpe (eds.), Sur les groupes hyperboliques d'après Mikhael Gromov, Progr. Math., vol. 83, Birkhäuser, Boston, MA, 1990.

[GS] S.M. Gersten and H.B. Short, Small cancellation theory and automatic groups, Invent. Math. 102 (1990), 305-334.

[G] M. Gromov, Hyperbolic groups, Essays in Group Theory (S.M. Gersten, ed.), Math. Sci. Res. Inst. Publ., vol. 8, Springer-Verlag, New York, 1985.

[M] L. Mosher, The geometry of cubulated 3-manifolds, Topology (to appear).

[OI] A. Yu. Ol'shanski, Hyperbolicity of groups with subquadratic isoperimetric inequality, Internat. J. Algebra Comput. 1 (1991), 281-289.

[P] P. Papasoglu, On the sub-quadratic isoperimetric inequality for groups, preprint, Columbia University, 1992.

[Sh] H. Short (ed.), Notes on word-hyperbolic groups, Group Theory from a Geometric Viewpoint, Proc. ICTP, Trieste (E. Ghys, A. Haefliger, and A. Verjovsky, eds.), World Scientific, Singapore, 1991.

Mathematics Department, Princeton University, Princeton, New Jersey 08544 Current address: The Mathematical Institute, 24-29 St. Giles, Oxford, OX1 3LB Great Britain E-mail address: bridson@math.princeton.edu 